\title{
Combination of endoscopic submucosal dissection and chemoradiation therapy for superficial esophageal squamous cell carcinoma with submucosal invasion
}

\author{
YOSUKE MOCHIZUKI $^{1}$, YASUHARU SAITO ${ }^{1}$, TOMOYUKI TSUJIKAWA ${ }^{2}$, \\ YOSHIHIDE FUJIYAMA $^{2}$ and AKIRA ANDOH ${ }^{3}$ \\ ${ }^{1}$ Division of Digestive Endoscopy; ${ }^{2}$ Department of Medicine; ${ }^{3}$ Division of Mucosal Immunology, \\ Graduate School of Medicine, Shiga University of Medical Science, Otsu, Japan
}

Received June 7, 2011; Accepted July 1, 2011

DOI: 10.3892/etm.2011.319

\begin{abstract}
The efficacy, safety and clinical outcomes of a combination of endoscopic submucosal dissection (ESD) with subsequent chemoradiation therapy (CRT) for superficial esophageal squamous cell carcinomas (superficial ESCC) remain unclear. We assessed the outcome of the combination of ESD plus CRT for superficial ESCC. Fourteen patients with superficial ESCC invading into the muscularis mucosa or submucosa were treated with ESD plus CRT from 2004 to 2010. En bloc resection of the lesion was successfully performed in all patients. The mean diameter of the lesions was $25 \mathrm{~mm}$ (range 10-55). The distribution of the depth of tumor invasion was to the muscularis mucosa in 8 patients, to the upper submucosal third (sm1) in 4 patients and to the middle submucosal third ( $\mathrm{sm} 2$ ) in 2 patients. The laterally resected margins and vascular invasion were cancer-negative in all patients, but lymph node involvement was detected in 2 patients. The mean follow-up period after CRT was 45 months (range 19-70). No patients died of esophageal cancer. Recurrence or metastasis of the esophageal cancer was not observed in any of the patients. The combination of ESD plus CRT is effective for superficial ESCC.
\end{abstract}

\section{Introduction}

The incidence of superficial esophageal squamous cell carcinoma (superficial ESCC) has increased due to advances in diagnostic modalities, including endoscopy and endoscopic ultrasonography. Because of the low risk of lymph node metastasis, endoscopic therapies, such as endoscopic mucosal resection (EMR) and endoscopic submucosal dissection (ESD),

Correspondence to: Professor Yasuharu Saito, Division of Digestive Endoscopy, Shiga University of Medical Science, Seta-Tukinowa, Otsu 520-2192, Japan

E-mail: ysaito@belle.shiga-med.ac.jp

Key words: esophageal cancer, endoscopic submucosal dissection, chemoradiation therapy have been established as standard therapeutic approaches for superficial ESCC within the lamina propria mucosa (1-4). On the other hand, due to a higher risk for lymph node metastasis, there remains controversy over which treatment should be employed for a superficial ESCC invading into the submucosal layer (5), even though endoscopic therapies are far less invasive as compared to radical surgery with lymphadenectomy. A recent study revealed an increasing rate of lymph node metastasis in superficial ESCC according to the infiltration level: $27 \%$ in the upper submucosal third (sm1), $36 \%$ in the middle submucosal third ( $\mathrm{sm} 2)$ and as high as $55 \%$ in the lower submucosal third (sm3) (5). In addition, estimates of the actual depth of tumor extension into the submucosal layer are sometimes difficult. Thus, in cases of superficial ESCC with submucosal invasion, the decision regarding endoscopic therapy vs. radical surgery requires weighing the benefits of the less invasive and less risky procedure against the potentially curative, although more invasive procedure, in patients with lymphatic involvement.

ESD is a recently developed procedure allowing accurate submucosal dissection and the en bloc resection of these mucosal lesions. Despite technical difficulties, ESD was shown to be an effective and relatively safe treatment for $\operatorname{ESCC}(4,6)$. When compared to EMR, ESD has been shown to reduce the local recurrence rate, as better lateral margin clearance can be achieved, thus allowing lesions with larger sizes to be resected with confidence $(4,6)$. However, the appropriateness of ESD should be carefully considered in the case of infiltration levels exceeding the lower mucosal third (m3), since previous studies have reported that the lymph node metastasis rates of ESCCs are significantly higher in cases of an infiltration level $\mathrm{m} 3$ as compared to infiltration levels of the upper (m1) and middle mucosal third (m2) (7). In Japan, ESD is generally restricted to esophageal cancers showing intramucosal invasion limited to the lamina propria mucosa, no vessel invasion, smaller than $2 \mathrm{~cm}$ in diameter and occupation of less than $2 / 3$ of the circumference of the lumen (4). Cases with infiltration over the mucosal layer are relatively indicated and require additional treatment, such as surgery. There are, however, few studies on the outcome of ESD with any additional treatments for superficial ESCC. 
We, therefore, conducted a retrospective analysis to investigate the efficacy, safety and clinical outcomes of the combination of ESD plus subsequent chemoradiation therapy (CRT) for superficial ESCC. The findings from this study provide useful information for determining the optimum treatment strategy for superficial esophageal cancers.

\section{Patients and methods}

Characteristics of the study population. From November 2004 to June 2010, 120 patients with superficial ESCC were treated by ESD at the Shiga University of Medical Science Hospital. Among the 120 patients, invasion to the muscularis mucosa or to the submucosal layer was pathologically observed in 18 patients and 14 of these received additional CRT instead of surgery. In these patients, CT scans did not detect any signs of lymph node or distant metastasis. The initial diagnosis of superficial esophageal cancer was made by high-resolution endoscopy with iodine staining, narrow-band imaging (8) and pathological examination of the biopsy samples. To evaluate the depth of tumor invasion into the submucosal layer, magnified endoscopy with narrow-band imaging and endoscopic ultrasonography (EUS) were used. Written informed consent was obtained from all patients. This study was approved by the Ethics Committee of the Shiga University of Medical Science.

Endoscopic submucosal dissection. ESD was performed with the patient under conscious sedation by the periodic intravenous administration of midazolam $(0.05-0.1 \mathrm{mg} / \mathrm{kg})$ and propofol $(2 \mathrm{mg} / \mathrm{kg} / \mathrm{h})$, while monitoring the blood pressure, heart rate and blood oxygen saturation. The endoscopes GIF-Q240, GIF-Q260 and GIF-Q260J (Olympus Optical Co., Ltd., Tokyo, Japan) were used. ESD was performed using a hook-knife (KD-620LR; Olympus) and an electrosurgical generator (VIO300D; ERBE Elektromedizin GmbH, Tuebingen, Germany). A transparent disposable attachment (Olympus) was fitted on the tip of the endoscope to obtain a constant view and tension on the connective tissue. The lateral margin of the lesion was determined by iodine staining and marking dots were made $\sim 2 \mathrm{~mm}$ outside of the boundaries of the lesions with the hook-knife. Next, a glycerin solution ( $10 \%$ in saline) containing $0.005 \mathrm{mg} / \mathrm{ml}$ epinephrine was injected into the submucosal layer to lift the lesion, which was incised outside the marking dots with the needle-knife (KD-1K-1; Olympus) using the Endocut mode of the electrosurgical generator. Subsequently, the thickened submucosal layer was dissected from the muscle layer using the hook-knife. Injections of glycerin solution were repeated on demand, and the submucosal layer was continuously dissected until all lesions were removed. To control bleeding, hemostatic forceps (FD-410LR; Olympus) were used in Soft coagulation mode.

Pathological examination. The dissected samples were evaluated pathologically according to the Japanese Classification of Esophageal Cancer: $\mathrm{m} 3$ was defined as a carcinoma that invaded into the muscularis mucosa; sm1 was defined as a carcinoma that infiltrated the submucosa (up to $200 \mu \mathrm{m}$ ) (below the lower border of the muscularis mucosa); and sm2 was defined as a carcinoma with deep invasion (more than a depth of $200 \mu \mathrm{m}$ ) in the submucosa. All specimens were examined microscopically to determine the histological type, depth of invasion, lateral and vertical resection margins, and presence of vascular involvement.

Chemoradiation therapy. CRT was initiated 2-4 weeks after ESD. External beam radiation therapy from the supraclavicular fossa to the esophagogastric junction, including the mediastinum, was performed. For patients with cancers located at the distal third of the esophagus, the radiation therapy field was extended to the cardia instead of the supraclavicular fossa. Radiation therapy was delivered 5 days per week at 2 Gy per fraction. The total dose was $40 \mathrm{~Gy}$ in 20 fractions over 4 weeks. The chemotherapy regimens consisted of 5-fluorouracil $\left(300-450 \mathrm{mg} / \mathrm{m}^{2}\right.$ of body surface area per day by 5 days of continuous intravenous infusion over 5 weeks) and cisplatin (30-40 $\mathrm{mg} / \mathrm{m}^{2}$ of body surface area per day by $2 \mathrm{~h}$ of intravenous infusion with hydration, on days 1, 8, 22 and 29).

Follow-up. Follow-up endoscopies were performed every 3-6 months in the first year after CRT, and every 6-12 months in the following years. In addition, CT scans of the neck, chest and abdomen were performed once a year to detect lymph node or distant metastasis.

\section{Results}

The features of the patients and their lesions are presented in Table I. A combination of ESD plus CRT was performed in 14 male patients (mean age 65 years; range 49-78). The localization of the lesion was as follows: 5 in the upper esophagus (36\%), 8 in the middle esophagus (57\%) and 1 in the lower esophagus (7\%). En bloc resection of the lesion was successfully performed in all patients. There were no complications with the ESD procedure. The mean diameter of the lesions was $25 \mathrm{~mm}$ (range 10-55). Two thirds of the circumference of the lumen was occupied by cancer in 1 patient, one half in 2 patients, one third in 2 patients, one quarter in 5 patients and one sixth in 4 patients. The distribution of the depth of tumor invasion was $\mathrm{mm}$ lesions which reached the muscularis mucosa in 8 patients (57\%), sm1 in 4 patients (24\%) and sm 2 in 2 patients (14\%). The laterally resected margins were cancer-negative in all patients and vascular invasion was also negative in all patients. Lymph node involvement was detected in 2 patients.

CRT was successfully performed in all patients. In 2 patients (14\%), the chemotherapy dose was reduced because of moderate hematologic toxicity (RTOG grade 2). Gastrointestinal symptoms, such as nausea and heartburn, appeared in 8 patients, but these symptoms were transient and disappeared rapidly. Other complications, such as radiation pneumonitis, esophageal fistulae and esophageal strictures, did not appear.

The mean follow-up period after CRT was 45 months (range 19-70). None of the patients died of esophageal cancer, but 2 patients died of other diseases $(1$ died of intestinal obstruction at 53 months after CRT and 1 of pharyngeal cancer progression at 19 months after CRT). With the exception of these 2 fatal cases, the mean follow-up period after CRT was 46.5 months. During the follow-up period, the recurrence of esophageal cancer was not observed in any of the patients. 
Table I. Patients with superficial esophageal squamous cell carcinoma.

\begin{tabular}{|c|c|c|c|c|c|c|c|c|c|c|}
\hline $\begin{array}{l}\text { Patient } \\
\text { no. }\end{array}$ & $\begin{array}{c}\text { Age } \\
\text { (years) }\end{array}$ & Gender & Location & Resection & $\begin{array}{l}\text { Size } \\
(\mathrm{mm})\end{array}$ & Circumference & $\begin{array}{l}\text { Depth of } \\
\text { invasion }\end{array}$ & $\begin{array}{l}\text { Resection } \\
\text { margin }\end{array}$ & $\begin{array}{l}\text { Lymphovascular } \\
\text { involvement }\end{array}$ & $\begin{array}{c}\text { Observed } \\
\text { period } \\
\text { (months) }\end{array}$ \\
\hline 1 & 74 & M & Lower & En bloc & $25 \times 20$ & $1 / 3$ & $\mathrm{~mm}$ & Free & ly0 v0 & 70 \\
\hline 2 & 53 & M & Middle & En bloc & $15 \times 15$ & $1 / 3$ & $\mathrm{~mm}$ & Free & ly0 v0 & 67 \\
\hline 3 & 63 & M & Upper & En bloc & $14 \times 10$ & $1 / 6$ & $\mathrm{~mm}$ & Free & ly0 v0 & 65 \\
\hline 4 & 61 & M & Middle & En bloc & $55 \times 30$ & $1 / 2$ & sm1 & Free & ly0 v0 & 58 \\
\hline 5 & 75 & M & Upper & En bloc & $48 \times 20$ & $2 / 3$ & $\mathrm{~mm}$ & Free & ly0 v0 & 59 \\
\hline 6 & 71 & M & Middle & En bloc & $24 \times 20$ & $1 / 4$ & $\operatorname{sm} 2$ & Free & ly0 v0 & 53 \\
\hline 7 & 60 & M & Upper & En bloc & $40 \times 38$ & $1 / 2$ & $\mathrm{~mm}$ & Free & ly0 v0 & 52 \\
\hline 8 & 51 & M & Middle & En bloc & $22 \times 20$ & $1 / 4$ & sm1 & Free & ly0 v0 & 27 \\
\hline 9 & 73 & M & Middle & En bloc & $30 \times 15$ & $1 / 4$ & $\mathrm{~mm}$ & Free & ly0 v0 & 3 \\
\hline 10 & 63 & M & Middle & En bloc & $10 \times 10$ & $1 / 6$ & $\mathrm{~mm}$ & Free & ly0 v0 & 33 \\
\hline 11 & 78 & M & Middle & En bloc & $25 \times 24$ & $1 / 4$ & $\mathrm{~mm}$ & Free & ly0 v0 & 23 \\
\hline 12 & 71 & M & Upper & En bloc & $20 \times 20$ & $1 / 6$ & sm1 & Free & ly0 v0 & 50 \\
\hline 13 & 49 & M & Upper & En bloc & $15 \times 15$ & $1 / 6$ & $\operatorname{sm} 2$ & Free & ly0 v1 & 20 \\
\hline 14 & 72 & M & Middle & En bloc & $20 \times 18$ & $1 / 4$ & sm1 & Free & ly0 v1 & 19 \\
\hline
\end{tabular}

$\mathrm{mm}$, lesions which reached the muscularis mucosa; sm 1 , a carcinoma that infiltrated the submucosa (up to $200 \mu \mathrm{m}$ ); sm 2 , a carcinoma with deep invasion into the submucosa (more than a depth of $200 \mu \mathrm{m}$ ).

Similarly, no clinical lymph node or distant metastasis was observed in any of the patients.

\section{Discussion}

Endoscopic therapies are recommended for superficial ESCC within the lamina propria mucosa. In contrast, CRT or surgery has been considered to be the standard therapeutic approach for ESCC with submucosal invasion. However, the mortality and postsurgical complications related to esophagectomy are not negligible. Based on this reason, radical surgery cannot be applied to all patients. For example, an esophagectomy may be difficult to perform in patients with advanced age, and cardiac or pulmonary complications. Such patients may be treated with CRT alone, but a definite cure cannot be expected. As one of the therapeutic options for superficial ESCC invading into the submucosal layer, we report here the usefulness of the combination of ESD plus CRT.

EMR has been shown to be a safer alternative, with longterm survival outcomes similar to those achieved with surgery. However, EMR is hampered by certain technical limitations, such as the low success rate of en bloc resection, in particular of lesions larger than $20 \mathrm{~mm}(4,7)$. Moreover, post-EMR recurrence or metachronous carcinoma was reported to occur in as many as $26 \%$ of these cases $(9,10)$. On the other hand, ESD enables the operator to achieve an en bloc resection regardless of the tumor size. Despite its technical difficulties, ESD was shown to be an effective and relatively safe treatment for squamous cell cancer of the esophagus (11). Thus, there are increasing numbers of reports showing that ESD is superior to conventional EMR as the treatment of choice for superficial ESCC. Takahashi et al (12) compared ESD ( $\mathrm{n}=116)$ against EMR ( $n=184)$ in 300 patients with superficial ESCC within the mucosa. The en bloc resection and the local recurrence rates were significantly better in the ESD group. They identified several risk factors for local recurrence and these were EMR, deep cancer invasion, an upper esophagus location and a family history of esophageal cancer. They experienced local recurrence ( $n=18$ in EMR and $n=1$ in ESD), lymph node metastasis $(n=4$ in EMR and $n=0$ in ESD) and distant metastasis ( $n=5$ in EMR and $n=0$ in ESD). They showed that the local and nodal recurrence rate was significantly higher in patients with an infiltration depth of $\mathrm{m} 3$ when compared to patients with sm1 or sm2. Katada et al analyzed the clinical outcome of 86 patients with superficial ESCC invading into the muscularis mucosa treated by EMR only (1). They experienced lymph node recurrence in 2 patients during a 43-month follow-up period. Teoh et al compared the efficacy of ESD against EMR in patients with ESCC within the mucosa (2). They concluded that ESD allows for the en bloc resection of larger mucosal lesions of the esophagus, which could not have been resected by EMR. Local recurrence was observed in 1 patient treated with EMR. These reports indicate that ESD is superior to EMR as a standard treatment for superficial ESCC. However, it should be emphasized that there are possible risks for local or lymph node recurrence in ESCC patients with $\mathrm{m} 3$ invasion treated by EMR or ESD, suggesting a much higher recurrence rate in ESCC invading into the submucosal layer, even when treated with ESD.

CRT is a useful tool for treating squamous-cell carcinoma of the esophagus. Previous studies have revealed that CRT resulted in outcomes comparable to those for surgical resection in patients with stage I or II cancers $(13,14)$. CRT plays a role in ESCC treatment via the control of local and distant recurrences. Previously, Murakami et al reported a 3 -year local control rate of $70 \%$ and a 3 -year survival rate of 
$83 \%$ in 24 patients with superficial esophageal carcinomas treated with CRT (14). They concluded that CRT resulted in comparable survival rates as conventional surgery for patients with superficial ESCC. Another Japanese multi-institutional study revealed that the 2-year local control rate was $83 \%$ in 105 patients with superficial ESCC treated with radiotherapy; 22 of these 105 patients had local recurrence within the radiation fields (15). Thus, previous studies have demonstrated sufficient efficacy for CRT in the control of local recurrence in patients with superficial ESCC.

None of the 14 patients who received CRT after ESD in the present study had local recurrences or metastases. None of the patients died of recurrent esophageal carcinomas or intercurrent diseases. Previously, the efficacy of a combination of EMR plus CRT for the treatment of superficial ESCC was reported (16). In this study, we employed ESD and evaluated the effects of a combination of ESD plus CRT. Collectively, the superiority of ESD has been demonstrated in many reports, and hence the combination of ESD plus CRT may be preferable. The overall and cause-specific survival rates in the ESD plus CRT group were favorable and ESD restored the quality of life (QOL) in these patients to pre-treatment levels. It is likely that the less invasive ESD plus CRT contributed not only to a better QOL, but also to better long-term outcomes by preserving physical strength.

The present study has several limitations, such as a retrospective single-center study design in a small number of cases. The use of control cases, such as monotherapy with ESD or CRT, may also be preferable. Future studies should be carried out using a larger number of cases and an extended follow-up period. Moreover, a prospective study involving a combination of ESD and unified CRT regimen should be performed to evaluate the recurrence and survival rate in relation to the degree of differentiation and the depth of invasion of superficial ESCC.

\section{References}

1. Katada C, Muto M, Momma K, et al: Clinical outcome after endoscopic mucosal resection for esophageal squamous cell carcinoma invading the muscularis mucosae - a multicenter retrospective cohort study. Endoscopy 39: 779-783, 2007.
2. Teoh AY, Chiu PW, Yu Ngo DK, Wong SK, Lau JY and Ng EK: Outcomes of endoscopic submucosal dissection versus endoscopic mucosal resection in management of superficial squamous esophageal neoplasms outside Japan. J Clin Gastroenterol 44: e190-e194, 2010.

3. Repici A, Hassan C, Carlino A, et al: Endoscopic submucosal dissection in patients with early esophageal squamous cell carcinoma: results from a prospective Western series. Gastrointest Endosc 71: 715-721, 2010

4. Fujishiro M, Yahagi N, Kakushima N, et al: Endoscopic submucosal dissection of esophageal squamous cell neoplasms. Clin Gastroenterol Hepatol 4: 688-694, 2006.

5. Gockel I, Sgourakis G and Lang H: Superficial esophageal cancer: endoscopic resection or radical surgery? Expert Rev Anticancer Ther 10: 1345-1347, 2010.

6. Ishihara R, Iishi H, Uedo N, et al: Comparison of EMR and endoscopic submucosal dissection for en bloc resection of early esophageal cancers in Japan. Gastrointest Endosc 68: 1066-1072, 2008.

7. Eguchi T, Nakanishi Y, Shimoda T, et al: Histopathological criteria for additional treatment after endoscopic mucosal resection for esophageal cancer: analysis of 464 surgically resected cases. Mod Pathol 19: 475-480, 2006.

8. Yoshida T, Inoue H, Usui S, Satodate H, Fukami N and Kudo SE: Narrow-band imaging system with magnifying endoscopy for superficial esophageal lesions. Gastrointest Endosc 59: 288-295, 2004.

9. Ishihara R, Iishi H, Takeuchi Y, et al: Local recurrence of large squamous-cell carcinoma of the esophagus after endoscopic resection. Gastrointest Endosc 67: 799-804, 2008.

10. Esaki M, Matsumoto T, Hirakawa K, et al: Risk factors for local recurrence of superficial esophageal cancer after treatment by endoscopic mucosal resection. Endoscopy 39: 41-45, 2007.

11. Oyama T, Tomori A, Hotta K, et al: Endoscopic submucosal dissection of early esophageal cancer. Clin Gastroenterol Hepatol 3: S67-S70, 2005.

12. Takashi H, Arimura Y, Masao H, et al: Endoscopic submucosal dissection is superior to conventional endoscopic resection as a curative treatment for early squamous cell carcinoma of the esophagus. Gastrointest Endosc 72: 255-264, 2010.

13. Bidoli P, Bajetta E, Stani SC, et al: Ten-year survival with chemotherapy and radiotherapy in patients with squamous cell carcinoma of the esophagus. Cancer 94: 352-361, 2002.

14. Murakami M, Kuroda Y, Nakajima T, et al: Comparison between chemoradiation protocol intended for organ preservation and conventional surgery for clinical T1-T2 esophageal carcinoma. Int J Radiat Oncol Biol Phys 45: 277-284, 1999.

15. Okawa T, Tanaka M, Kita-Okawa M, et al: Superficial esophageal cancer: multicenter analysis of results of definitive radiation therapy in Japan. Radiology 196: 271-274, 1995.

16. Shimizu Y, Kato M, Yamamoto J, et al: EMR combined with chemoradiotherapy: a novel treatment for superficial esophageal squamous-cell carcinoma. Gastrointest Endosc 59: 199-204, 2004. 